\title{
Hyperglycemia Caused Damage to Mitochondria on Early Stage of Reperfusion Following A Transient Cerebral Ischemia
}

\author{
Y. Lin, ${ }^{*}$ Q.P. He, ${ }^{* *}$ M. Muranyi, ${ }^{*}$ and P.A. Li* \\ * Department of Cell and Molecular Biology and Medicine, University of Hawaii School \\ of Medicine, Honolulu, HI 96822 \\ **Biological Electron Microscope Facility, University of Hawaii, Honolulu, HI 96822
}

\begin{abstract}
Although hyperglycemia caused either by glucose infusion or diabetes exaggerates brain damage induced by transient focal and global ischemia, the mechanisms of hyperglycemia-exaggerated damage have not been defined. The aim of current study was to determine whether hyperglycemia causes damage to the mitochondria in early stage of reperfusion following a transient global cerebral ischemia in rats.
\end{abstract}

Two length of global cerebral ischemia were induced in Wistar rats. One is $30 \mathrm{~min}$ ischemia followed by 60 min of recovery in normoglycemic and acute glucose infusion induced hyperglycemic rats, Brains in these rats were fixed with $4 \%$ glutaraldehyde in $0.1 \mathrm{M}$ cacodylate buffer ( $\mathrm{pH} 7.4$ ). The other is $15 \mathrm{~min}$ ischemia followed by $3 \mathrm{hrs}$ reperfusion in sham operated and glucose infusion-induced hyperglycemic rats. The mitochondria were isolated using Percoll gradient method. Both samples were fixed with $4 \%$ glutaraldehyde and processed for electron microscopic examination. The results showed that rats subjected to $30 \mathrm{~min}$ ischemia and $60 \mathrm{~min}$ reperfusion, the nucleus was relatively homogenous, the nucleolus was clearly visible, and mitochondrial morphology was normal in normoglycemic animals. In contrast, marked chromatin condensation, severe vacuolization and evident mitochondrial swelling, lucency, and loss of cristae were the features of hyperglycemic rats [2]. No apoptotic bodies were observed in any of the ultrathin sections examined. In agreement with the findings, mitochondria isolated after $3 \mathrm{hrs}$ of reperfusion following a $15 \mathrm{~min}$ ischemia showed severe swelling while the mitochondrial isolated from sham-operated control rats displayed predominantly normal morphology.

The results suggest that pre-existing hyperglycemia augments ischemic brain damage by causing mitochondrial damage in early stage of reperfusion. This has been further supported by our studies showing that hyperglycemia causes early release of cytochrome $\mathrm{c}$ from the mitochondria to the cytoplasm and activates a mitohcondria-initiated cell death pathway after ischemia and reperfusion [1-3]

\section{References}

[1] C. Ding et al., Exp Neurol. 188 (2004) 421.

[2] P.A. Li et al., J Cereb Blood Flow Metab. 21 (2001) 568.

[3] M. Muranyi et al., Diabetes. 52 (2003) 481.

[4] This study was supported by NIH/NCRR (RR16453, RR03061) and Ingeborg v. F. Mckee Fund of Hawaii Community Foundation to PAL. 

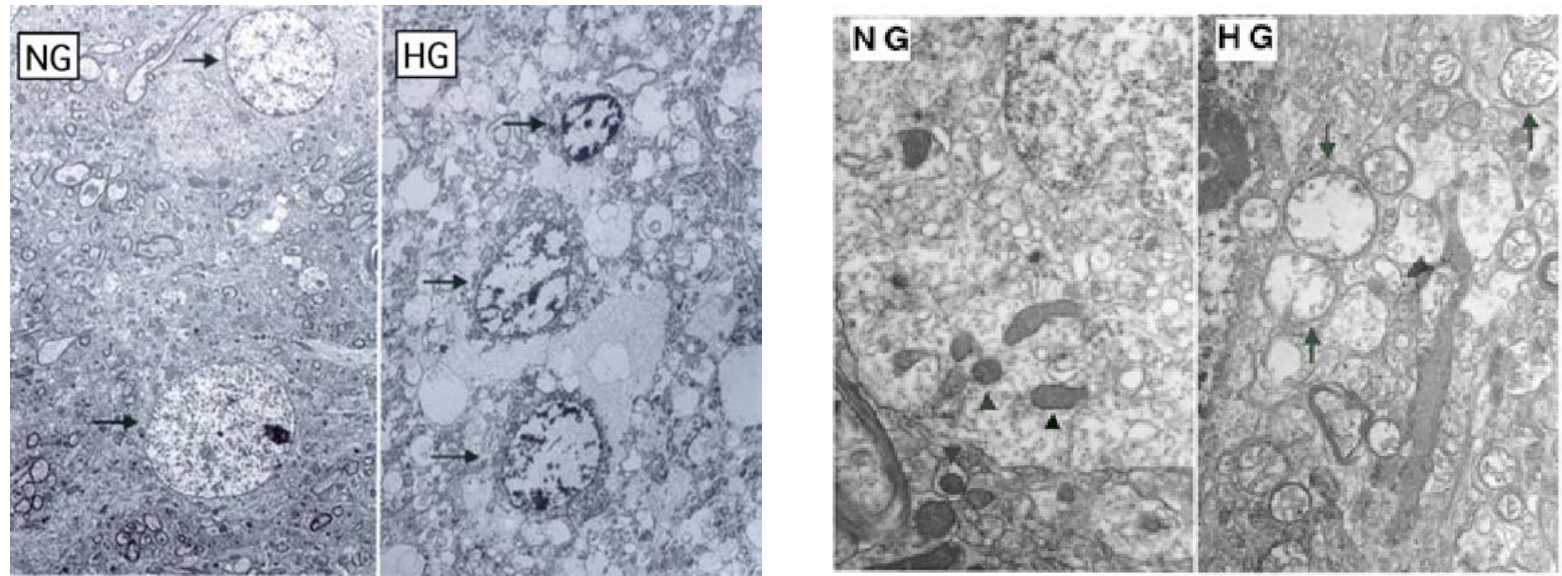

Figure 1. Nuclear and mitochondrial ultrastructural changes after cerebral ischemia and reperfusion in the cortex. $\mathrm{NG}$ : normoglycemia; $\mathrm{HG}$ :

hyperglycemia. Arrows heads in the left panels point to nuclei and arrows in the right panel indicate swelling mitochondria. Arrowheads indicate normal mitochondria. Magnifications for left and right panels were 2,000X and 10,000X, resp Fig. 10. Ultrastructural changes of mitochondria Isolated from cortices of normal (Left) and ischemic (Right) rats with HG condition after $3 \mathrm{hrs}$ of recovery. Arrows denote morphologically impact mitochondria and * denote swollen mitochondria. Bar $=1 \mu \mathrm{m}$.

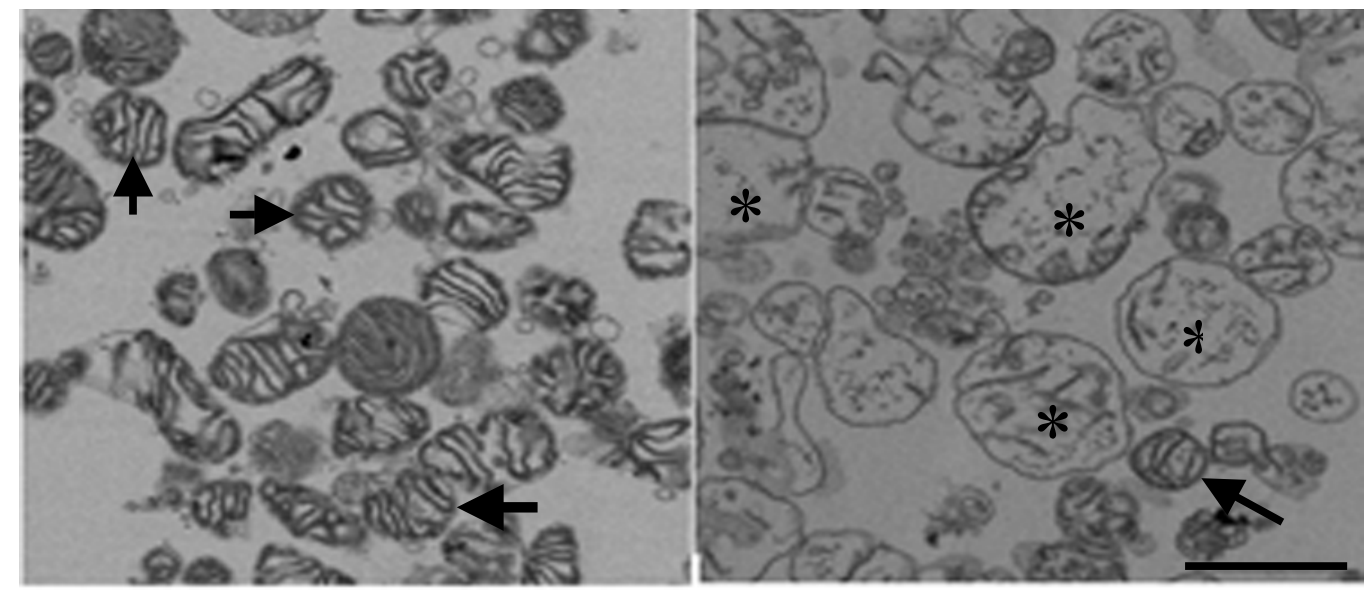

Figure 2. Ultrastructural changes of mitochondria Isolated from cortices of normal (Left) and ischemic (Right) rats with HG condition after 3 hrs of recovery. Arrows denote morphologically impact mitochondria and * denote swollen mitochondria. Bar $=1 \mu \mathrm{m}$. 